\title{
Different Muscle Action Training Protocols on Quadriceps- Hamstrings Neuromuscular Adaptations
}

\author{
Authors \\ Cassio V. Ruas ${ }^{1,4}$, Lee E. Brown ${ }^{2}$, Camila D. Lima3 ${ }^{3}$ G. Gregory Haff', Ronei S. Pinto ${ }^{4}$
}

\section{Affiliations}

1 Centre for Exercise and Sports Science Research (CESSR), School of Medical and Health Sciences, Edith Cowan University, Joondalup, Australia

2 Department of Kinesiology, California State University Fullerton, Fullerton, United States

3 School of Human Kinetics and Recreation, Memorial University of Newfoundland Saint John's, Canada

4 Exercise Research Laboratory, Department of Physical Education, Physiotherapy and Dance, Federal University of Rio Grande do Sul, Porto Alegre, Brazil

Key words

concentric, eccentric, short-term resistance training

accepted after revision 03.01.2018

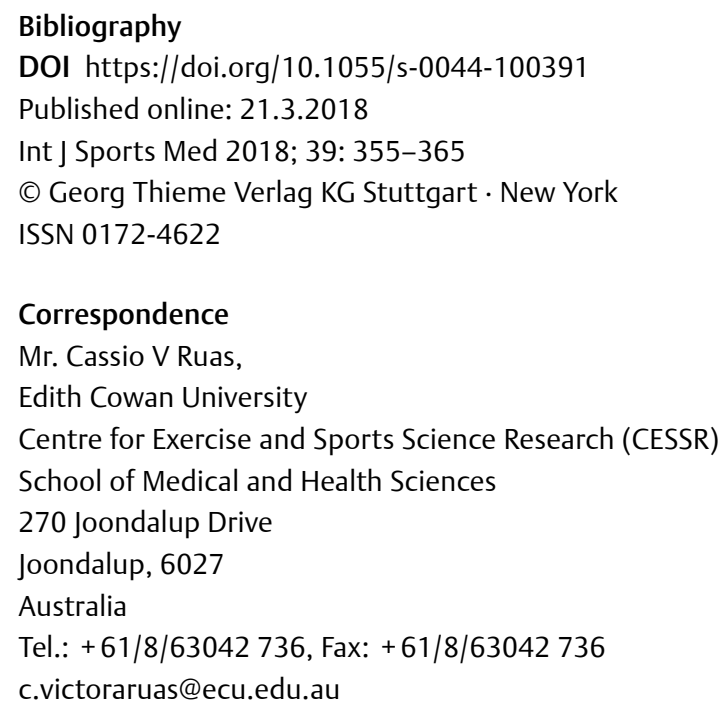

\begin{abstract}
The aim of this study was to compare three specific concentric and eccentric muscle action training protocols on quadricepshamstrings neuromuscular adaptations. Forty male volunteers performed 6 weeks of training (two sessions/week) of their dominant and non-dominant legs on an isokinetic dynamometer. They were randomly assigned to one of four groups; concentric quadriceps and concentric hamstrings (CON/CON, $n=10$ ), eccentric quadriceps and eccentric hamstrings (ECC/ ECC, $n=10$ ), concentric quadriceps and eccentric hamstrings (CON/ECC, $n=10$ ), or no training (CTRL, $n=10)$. Intensity of training was increased every week by decreasing the angular velocity for concentric and increasing it for eccentric groups in $30 \%$ increments. Volume of training was increased by adding one set every week. Dominant leg quadriceps and hamstrings muscle thickness, muscle quality, muscle activation, muscle coactivation, and electromechanical delay were tested before and after training. Results revealed that all training groups similarly increased MT of quadriceps and hamstrings compared to control $(p<0.05)$. However, CON/ECC and ECC/ECC training elicited a greater magnitude of change. There were no significant differences between groups for all other neuromuscular variables $(p>0.05)$. These findings suggest that different shortterm muscle action isokinetic training protocols elicit similar muscle size increases in hamstrings and quadriceps, but not for other neuromuscular variables. Nevertheless, effect sizes indicate that CON/ECC and ECC/ECC may elicit the greatest magnitude of change in muscle hypertrophy.
\end{abstract}

\section{Introduction}

The mechanisms underlying strength increases following resistance training (RT) have been associated with neuromuscular adaptations [24,47]. Neural adaptations can be interpreted as increases in the level of efferent neural output from the central nervous system to activated muscle fibers, which may be facilitated by increased activation of agonist and reduced coactivation of antagonist muscles [20]. Muscular adaptations may occur due to muscle architectural and molecular changes induced by resistance training, which are reflected in strength gains [45]. Although a high level of mechanical loading has been shown to maximally recruit higher threshold motor units, as well as optimizing neural activation and muscle fiber growth [35], specific muscle action training may lead to distinct neuromuscular adaptations [24, 39, 43,47]. Concentric and eccentric induced adaptations may depend on the specific muscle action trained and tested, due to their different mechani- 
cal efficiency, force output and force/velocity relationships [19,47]. However, contradictory results have been found in the scientific literature regarding the time course of neurological (e.g. muscle activation) and morphological adaptations (e. g. muscle size) to resistance training as a result of intensity, volume, frequency, length of training, and mode of training $[2,46,50]$.

Although muscle activation (MA) and muscle thickness (MT) have been previously investigated following concentric and/or eccentric RT [2, 3, 6, 19, 24, 39, 47], little is known about other variables that contribute to neuromuscular adaptations, such as muscle quality (MQ), muscle coactivation (MCA) and electromechanical delay (EMD). Concentric and eccentric training have been shown to similarly increase quadriceps MQ measured by reducing the muscle's echo-intensity, which indirectly reflects the level of a muscle's intramuscular fat and/or fibrous composition [6, 37, 44]. Resistance training may lead to an increased muscle contractile component resulting in reduced intramuscular fat and/or fibrous composition levels [18]. Additionally, eccentric exercise has been associated with a high reduction in muscular co-contraction [40], which is believed to be related to improved agonist muscle neural drive [28]. Finally, RT individuals, when compared to endurance athletes, have been found to have shorter EMD [53], which represents the time between the onset of electrical activity at the muscle and the onset of torque, and is positively related to stiffness changes in the serieselastic component [10, 30, 31]. Nevertheless, RT involving concentric and eccentric muscle actions have not been found to be effective in decreasing EMD [14, 22].

Very few studies have compared neuromuscular adaptations after quadriceps and hamstrings concentric (CON/CON) or eccentric (ECC/ECC) muscle action training protocols $[6,24,47]$, and no studies, to our knowledge, have investigated how these adaptations differ from concentric (agonist) and eccentric (antagonist) (CON/ECC) combined training. Also, although unique neuromuscular characteristics of specific muscle action training are believed to influence these outcomes, it is questionable whether these differences occur after short-term RT $[11,50]$. Therefore, the aim of this study was to compare six weeks of CON/CON, ECC/ECC and CON/ECC muscle action training on MT, MQ, MA, MCA, and EMD of hamstrings and quadriceps. Our hypothesis was that ECC/ECC and CON/ECC training would lead to greater increases in all neuromuscular variables compared to CON/CON, because of their eccentric component, which present unique neuromuscular characteristics allowing for greater force production per cross bridge or series elastic component $[24,47]$. The results of the present study provide greater insight into the neuromuscular adaptations underpinning different resistance training based on concentric and eccentric muscle actions.

\section{Materials and Methods}

\section{Participants}

Sample size calculation was performed by G * Power 3.1 (Institute for Experimental Psychology, Dusseldorf, Germany) based on effect size of 0.4 , alpha level of 0.05 , and power of 0.90 . Effect size based on studies comparing concentric vs. eccentric resistance training on muscle thickness and muscle activation [24, 47]. By using this effect size, the sample size of the present study was calculated to be 36 , but 40 participants were recruited to avoid potential risk of dropouts throughout the study. Forty male volunteers (age $22.9 \pm 2.3$ years, mass $70.7 \pm 11.0 \mathrm{~kg}$, ht $174.3 \pm 6.9 \mathrm{~cm}$ ), who were not involved in any strength or endurance training programs for the previous 3 months were recruited for participation in the present study. All participants were free of any knee injuries, and had refrained from physical activity $48 \mathrm{~h}$ prior to the first testing session. Prior to testing, they were asked for their leg preference for kicking (dominance) [33]. All participants read and signed a University Institutional Review Board-approved informed consent form based on the Declaration of Helsinki prior to participation. In the consent form, participants completed sections about health and safety conditions, which included having abstained from alcohol and caffeine $12 \mathrm{~h}$ prior to pre- and post-training sessions, and prior to each training session. The present study meets the ethical standards in sports and exercise science research [23].

\section{Experimental design}

Participants were randomly assigned to one of four groups; CON/ CON ( $n=10$, age $22.0 \pm 1.5$ years, mass $73.6 \pm 9.9 \mathrm{~kg}$, ht $175.2 \pm 7.3 \mathrm{~cm}), E C C / E C C(n=10$, age $22.9 \pm 2.3$ years, mass $73.9 \pm 12.7 \mathrm{~kg}$, ht $175.8 \pm 7.9 \mathrm{~cm})$, CON $/$ ECC $(n=10$, age $23.4 \pm 2.6$ years, mass $70.2 \pm 12.1 \mathrm{~kg}$, ht $175.5 \pm 7.0 \mathrm{~cm}$ ), or no training (CTRL, $n=10$, age $23.2 \pm 2.6$ years, mass $64.8 \pm 7.9 \mathrm{~kg}$, ht $170.6 \pm 4.5 \mathrm{~cm}$ ). Training consisted of six weeks of dominant and non-dominant knee exercise on a Biodex isokinetic dynamometer. Dominant leg quadriceps and hamstrings MT, MQ, MA, MCA, and EMD were tested before and after training. All dependent variables have been previously shown to be important indicators of neuromuscular adaptations related to concentric and/or eccentric RT $[2,3,6,19,24,39,47]$.

\section{Procedures}

\section{Pre-training tests}

Pre-training tests were performed on one day, $72 \mathrm{~h}$ before training. Participants were first measured for mass on a digital scale (model \# ES200L, Ohaus, Pine Brook, NJ, USA) and for height on a wallmounted stadiometer (Seca Stadiometer, Ontario, Canada). They were then measured for ultrasound $\mathrm{MT}$ and MQ, followed by isometric maximal testing, including MA and MCA, as well as EMD measurements.

\section{Ultrasound measurements}

Participants laid in a supine position for 10 min with both arms and legs extended and relaxed in order to stabilize normal body fluids $[38,44]$. Quadriceps images were measured first before hamstrings in the prone position. These positions ensured that the legs were extended and muscles relaxed during all measurements [8]. MT and MQ measurements were then made by three consecutive images in the transverse plan of the quadriceps rectus femoris, vastus intermedius, vastus lateralis and vastus medialis, and hamstrings biceps femoris long head, semitendinosus and semimembranosus muscles of the dominant leg using a real-time portable B-mode ultrasound device (GE LOGIQTM e, GE Healthcare, WI, USA) 
with linear-array transducer (code 12L-RS, variable frequency band 4.2-13.0 Mhz). In order to ensure acoustic contact and avoid risk of increased contact pressure, a water-based gel was used between the skin and transducer [8]. All measurements were at $50 \%$ of the distance between the lateral condyle and greater trochanter of the femur, except for the vastus medialis which was at $30 \%$ [38]. Although muscle size changes could occur at other sites in the hamstrings, this anatomical site was chosen since it has previously been shown to have a strong correlation to cross sectional area measured by magnetic resonance imaging [1]. The transducer was placed perpendicular to the muscle fibers and transparency film was used to map the skin to ensure measurements matched between days [44]. The same settings for gain (52 dB), depth $(9 \mathrm{~cm})$, and frequency $(12 \mathrm{MHz}$ ) were used in all measurements to optimize quality $[36,44]$.

MT values were measured at the widest distance between the adipose muscle upper interface and the lower interface, except for vastus intermedius, which was measured at the widest distance between the muscle upper interface and the bone [36, 38, 44]. Distances were measured using the device straight-line function, and the average of three MT measurements was calculated for each muscle, using Image] software (Version 1.48v, National Institutes of Health, Bethesda, MD, USA). MT measurements for pre- and post-training tests of all muscles presented a maximal coefficient of variation $\leq 21.12 \%$.

MQ was measured through echo-intensity values of each muscle by using the polygon function, surrounding the muscles without including fascia or bone, and grayscale analyses were performed using the histogram function of the Image] software. Echointensity values were expressed in a range between 0 and 255 , where black $=0$ and white $=255[8,44]$. The average of three echointensity measurements was calculated for each muscle. Echo-intensity from the vastus intermedius may have been affected by echo beam attenuation as this was the deepest muscle measured [51]. El measurements for pre- and post-training tests of all muscles presented maximal coefficient of variation $\leq 4.39 \%$.

All images were saved and exported to Imagej software for analyses. An experienced researcher in ultrasound assessments performed all measurements. The first ten participants visited the lab one day before commencement of the study. They performed ultrasound measurements on two consecutive days to calculate testretest reliability for $\mathrm{MT}$ and MQ. Intraclass correlation coefficients (ICC) $(1,1)$ [49], standard error of measurement (SEM), minimum difference to be considered real (MD), coefficient of variation (CV\%) and $95 \%$ lower bound and upper bound confidence intervals ( $\mathrm{LBCl}$ and $\mathrm{UBCl}$, respectively) were measured for MT and MQ. Quadriceps MT and MQ were in a range of 0.92-0.99 and 0.74-0.93 (ICC); 0.701.85 and 1.55-3.10 (SEM); 1.93-5.13 and 4.31-8.59 (MD); 2.586.05 and 4.60-8.58 (CV\%); 0.68-0.96 and 0.02-0.72 (LBCI); and $0.98-1.00$ and $0.97-0.98(\mathrm{UBCl})$. Hamstrings MT and MQ were in a range of 0.81-0.99 and 0.82-0.93 (ICC); 0.92-2.39 and 3.62-3.93 (SEM); 2.54-6.64 and 10.29-10.90 (MD); 2.36-6.05 and 9.02-9.27 (CV\%); 0.24-0.97 and 0.29-0.74 (LBCI); and 0.95-1.00 and 0.960.98 (UBCl) for hamstrings muscles $(p<0.05)$. According to Munro [32] these results are in a range considered as highly reliable.

\section{MA, MCA and EMD}

$M A, M C A$ and EMD were measured during maximal quadriceps and hamstrings isometric tests on the Biodex isokinetic dynamometer. Participants sat on the seat at $85^{\circ}$ of hip flexion and had straps applied across their thighs, waist and chest. The dynamometer's axis of rotation was aligned with the lateral femoral condyle and the lower leg was attached to the lever arm with the ankle cuff slightly above the medial malleolus [5]. Before testing, participants performed an isokinetic concentric extension-flexion warm-up of 10 repetitions at $180^{\circ} / \mathrm{s}$ through $90^{\circ}$ of range of motion $\left(0^{\circ}\right.$ at full extension). Their leg was then positioned at $60^{\circ}$ of knee extension for the isometric test. Testing began with quadriceps followed by hamstrings. Both tests consisted of three repetitions of $5 \mathrm{~s}$, and were separated by $5 \mathrm{~min}$ of rest. They were asked to push for the quadriceps isometric test and to pull for the hamstrings isometric test as hard as possible. Participants performed submaximal preliminary isometric repetitions before each test for familiarization purposes [5]. Verbal encouragement was provided during all tests, but no visual feedback was given.

During the isometric maximal test, participants were fitted with electrodes in order to measure quadriceps rectus femoris, vastus lateralis and vastus medialis, and hamstrings biceps femoris long head MA and MCA. The same anatomical sites used for ultrasound measurements were used for placement of the electrodes on the skin, using 4 bipolar ( $3.5 \mathrm{~cm}$ center-to-center) disposable surface electrodes (EL500 silver-silver chloride; BIOPAC Systems, Inc., Goleta, CA, USA). The area was shaved, abraded and cleaned with an isopropyl alcohol pad. Electrodes were replaced every time there were high levels of noise, which may be indicative of high skin impedance [15]. EMG signals were filtered by fourth-order Butterworth, $10-500 \mathrm{~Hz}$, as well as preamplified using a Myopac EMG device (MPRD-101; Run Technologies, Mission Viejo, CA, USA) bandwidth $=1-500 \mathrm{~Hz}$ amplifier with sampling frequency of $1000 \mathrm{~Hz}$. Root mean square (RMS) was calculated over a $1 \mathrm{~s}$ plateau of the curve during each knee extension and flexion maximal voluntary isometric contraction. This time interval was determined as soon as the maximal plateau of the curve was attained via a custom LabVIEW (version 2014 National Instruments Corporation, Austin, TX, USA). The average of three values was used for further analyses. MA and MCA measurements for pre- and post-training tests of all muscles presented maximal coefficient of variation values of $\leq 15.26 \%$ and $20.62 \%$, respectively.

EMD was calculated as the time delta between EMG start and torque start $[10,31]$ using the LabVIEW software with sampling at $1000 \mathrm{~Hz}$. The EMG start was defined as the point at which the RMS signal reached 3 standard deviations (SDs) above baseline, while the torque start was defined as the point at which the signal was above zero $\mathrm{Nm}$ [48]. The muscle representing quadriceps was vastus lateralis, while the muscle representing hamstrings was biceps femoris long head. The average of three EMD values of each muscle was used for further analyses. EMD measurements for pre- and post-training tests of all muscles presented maximal coefficient of variation $\leq 15.26 \%$.

All variables tested have been shown to have medium to high reliability in previous studies using similar procedures $[17,26,27]$. 


\section{Training sessions}

Training sessions were performed two times a week for 6 weeks. Each session lasted approximately $20 \mathrm{~min}$. The first training session began $72 \mathrm{~h}$ after pre-training tests and subsequent sessions were separated by at least $48 \mathrm{~h}$. Participants sat on a Biodex System 3 isokinetic dynamometer using the same procedures as the isometric test. Group CON/CON began by performing one set of 10 maximal repetitions at $210 \%$ for quadriceps and hamstrings; group ECC/ECC began by performing one set of 10 maximal repetitions at $60 \%$ for quadriceps and hamstrings; and group CON/ECC began by performing one set of 10 maximal repetitions at $210 \%$ for quadriceps concentric and at $60 \% \mathrm{~s}$ for hamstrings eccentric. Intensity of training was increased every week by decreasing isokinetic angular velocity for concentric and increasing it for eccentric groups in $30 \%$ increments $[21,29]$. Volume was increased by adding one set each week $[2,6]$. Group CTRL did not do any training, but returned to the lab for testing 6 weeks later.

\section{Post-training tests}

Post-tests were performed on one day $72 \mathrm{~h}$ after the last training session. It is unlikely that participants had high levels of muscle damage between the last session of eccentric training and the ultrasound assessment, as this mode of training is expected to induce the repeated bout effect over time [34]. This time interval has been previously found to be sufficient to reduce muscle damage markers (e. g. muscle swelling) [52], which could potentially affect ultrasound measures. In addition, participants did not report signs of exercise-induced muscle damage in any group. Participants performed the same tests as pre-training in the same order.

\section{Statistical analyses}

Normality of all values was verified by the Shapiro-Wilk test. Two $7 \times 2 \times 4$ (muscle $x$ time $x$ group) repeated measures ANOVAs were used to compare MT and MQ. Two $4 \times 2 \times 4$ (muscle $x$ time $x$ group) ANOVAs were used to compare MA and MCA. A $2 \times 2 \times 4$ (muscle $x$ time $x$ group) ANOVA was used to compare EMD. All analyses were performed with SPSS 21.0 (Statistical Package for Social Sciences, Chicago, IL, USA). Interactions and main effects were followed up by paired t-tests and post-hoc (LSD) analyses. An a priori alpha level of 0.05 was used to determine statistical significance. Data are expressed as mean and SD. Effect sizes for each significant difference between pre- and post-test comparisons by group were calculated by Cohens $\mathrm{d}$, in which values $<0.50$ were considered trivial, $0.50-$ 1.25 small, 1.25-1.9 moderate, and > 2.0 large for untrained participants [41]. Rhea [41] advocates this use along with the scale to determine effect sizes in resistance training research as a more applicable method to provide the magnitude of the resistance training intervention to exercise science professionals.

\section{Results}

For MT there were significant interactions of muscle $x$ time $(F=2.34$; $p=0.033)$ and time $x$ group $(F=7.65 ; p=0.00040)$. They were followed up with seven paired t-tests, one for each muscle, and two paired t-tests, one for each group. Vastus lateralis, vastus medialis, semitendinosus and semimembranosus post were greater than pre $(p<0.05)$, while rectus femoris and vastus intermedius post were not different than pre $(p>0.05)$. For groups CON/CON, ECC/ECC and CON/ECC post was greater than pre $(p<0.05)$. For group CTRL post was not different than pre $(p>0.05)$. Groups CON/ECC and ECC/ECC had moderate effect sizes, followed by CON/CON and CTRL, with small and trivial effect sizes, respectively ( $\vee$ Table $\mathbf{1}$ ).

For MQ, there were no interactions. However, there was a main effect for time $(F=9.047 ; p=0.0047)$, where pre was greater than post. There was also a main effect for muscle $(F=41.52$; $p<0.0001)$ where rectus femoris was greater than vastus intermedius and vastus medialis. Vastus lateralis was greater than vastus intermedius and vastus medialis. Vastus medialis was greater than vastus intermedius. Biceps femoris long head was greater than rectus femoris, vastus intermedius, vastus lateralis and vastus medialis. Semimembranosus was greater than rectus femoris, vastus intermedius, vastus lateralis and vastus medialis. Semitendinosus was greater than rectus femoris, vastus intermedius, vastus lateralis, vastus medialis and biceps femoris long head. ( $\triangleright$ Table 2 ).

For MA, there were no interactions. However, there was a main effect for time $(F=6.42 ; p=0.015)$, where post was greater than pre, and a main effect for muscle $(F=7.30 ; p=0.00058)$, where rectus femoris was greater than vastus lateralis, vastus medialis and biceps femoris long head ( $\mathbf{F i g} \mathbf{1}$ ).

For MCA, there was an interaction of muscle $x$ time $(F=80.695$; $p<0.0001)$. This was followed up with four paired t-tests, one for each muscle. Biceps femoris long head post was greater than pre $(\mathrm{p}<0.05)$ ( $\vee$ Fig. 2).

For EMD, there was an interaction of muscle $x$ time $(F=4.62$; $p=0.038)$. This was followed up with two paired t-tests, one for each muscle. Quadriceps pre was greater than post ( $p<0.05)$. Hamstrings pre was not different than post $(p>0.05)$ ( $\triangleright$ Fig. 3).

Individual data of MA, MCA and EMD between groups are presented in > Fig. 4-6.

\section{Discussion}

The aim of this study was to compare six weeks of CON/CON, ECC/ ECC and CON/ECC muscle action isokinetic training on MT, MQ, MA, MCA, and EMD of the hamstrings and quadriceps. Our results revealed that all training groups increased MT of both the quadriceps and hamstrings when compared to CTRL. However, effect sizes demonstrated that CON/ECC and ECC/ECC had a greater magnitude of change compared to CON/CON and CTRL. There were no differences between groups for MQ, MA, MCA and EMD. These findings suggest that all training protocols elicit muscle size increases, but the addition of eccentric training may elicit a greater MT magnitude. However, different short-term muscle action isokinetic training does not lead to differential adaptations in MA, MCA, MQ and EMD.

A plethora of studies have investigated MT following specific concentric and eccentric muscle action training modes [2, 3 , $6,19,24,39,47]$. Since maximal force output, mechanical efficiency and force-velocity relationships are different between distinct muscle actions, different muscle action training modes have been suggested to lead to specific morphological changes [19, 24, 47]. 


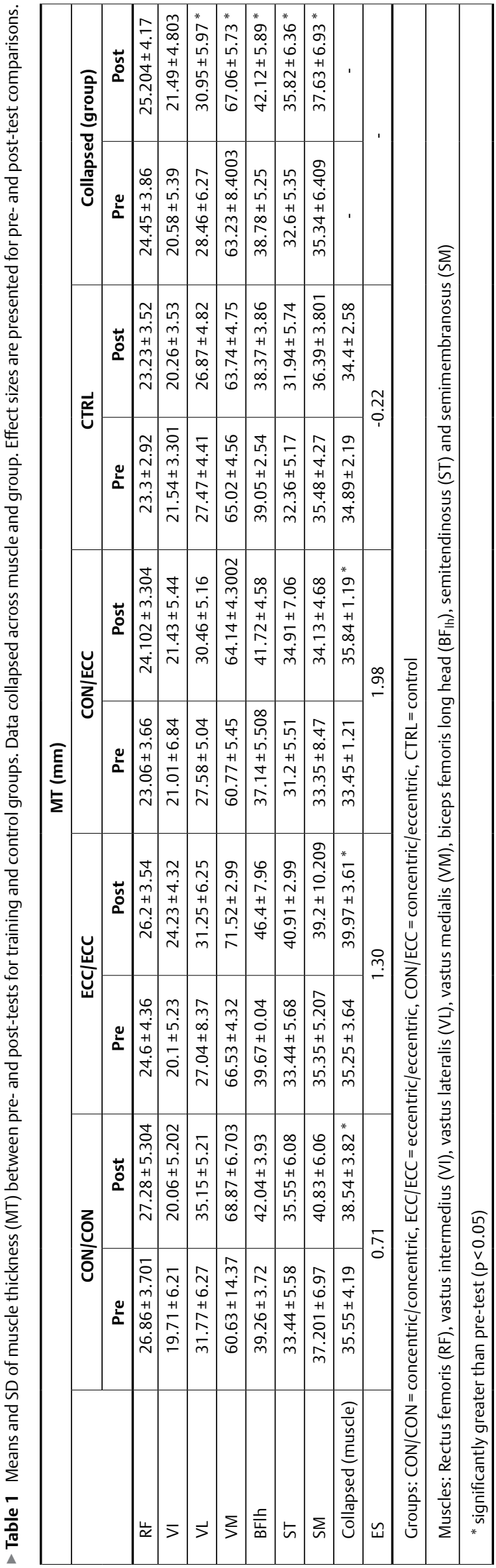

However, Werborn et al. [50], in a review study, concluded that there is not enough evidence at present to support superiority of any muscle action training mode for increasing muscle size in the short-term. This is in agreement with previous studies comparing concentric vs. eccentric training on muscle size adaptations $[3,6,19]$. Franchi et al. [19] found that 10 weeks of concentric vs. eccentric training of the knee extensors resulted in similar vastus lateralis hypertrophy adaptations for both modes. However, eccentric training led to a greater fascicle length (addition of sarcomeres in series), while concentric training led to a greater pennation angle (addition of sarcomeres in parallel), which suggests that the distribution of muscle hypertrophy along the vastus lateralis is highly dependent on the muscle action trained. Contrary to these findings, Blazevich et al. [3], found that vastus lateralis fascicle length increased similarly in concentric and eccentric training after 5 weeks of a 10-week training program, and concluded that muscle architecture adaptations occur very early in response to resistance training, but are not strongly influenced by any specific contraction mode. Although we did not measure architectural changes, our results are in agreement with these studies since all training modes increased MT similarly compared to CTRL.

Although our results demonstrated similar significant increases in MT, effect sizes demonstrated that CON/ECC and ECC/ECC elicited the greatest magnitude. Higbie et al. [24] found that although quadriceps cross sectional area increased after 10 weeks for both eccentric and concentric training, eccentric training led to slighter greater muscle size gains. Seger et al. [47] also demonstrated slightly greater quadriceps hypertrophy after 10 weeks of eccentric vs. concentric training. This is partially in accordance to our results as, although slight, these increases were significant. A greater number of participants per group in our study could have led our eccentrically trained groups to reach significantly greater MT results compared to CON/CON. Additionally, very short periods of up to 4 weeks of eccentric RT have previously been shown to be effective at increasing quadriceps MT [2]. Therefore, the eccentric component of our CON/ECC and ECC/ECC training may explain the greater MT magnitude compared to CON/CON, since the force demand in muscular connective tissues in lengthening actions is greater than in shortening, which may elicit greater mechanical stress, resulting in a greater stimulus for muscle size increases $[24,47]$. In addition, unaccustomed maximal eccentric exercise has been shown to lead to high levels of muscle damage and consequent alterations in muscular connective tissues [16]. This may induce greater sensitivity and stimulation of signaling pathways by transmembrane receptors, such as integrins, that promote adhesion of myofibers to muscular connective tissues and are pathway regulators for muscle fiber hypertrophy [16]. However, in our previous study comparing these same groups over 6 weeks of resistance training, we found that ECC/ECC resistance training only increased eccentric and isometric peak torque compared to CON/ $\mathrm{ECC}$ and $\mathrm{CON} / \mathrm{CON}$ training [42]. This questions whether neural or molecular factors rather than mechanical stress are the main contributors to greater strength increases in eccentrically only trained groups, which is an alternative explanation suggested by previous studies $[12,39]$.

Traditional concepts of RT suggest that neural adaptations occur earlier than morphological changes [50]. However, recent investi- 


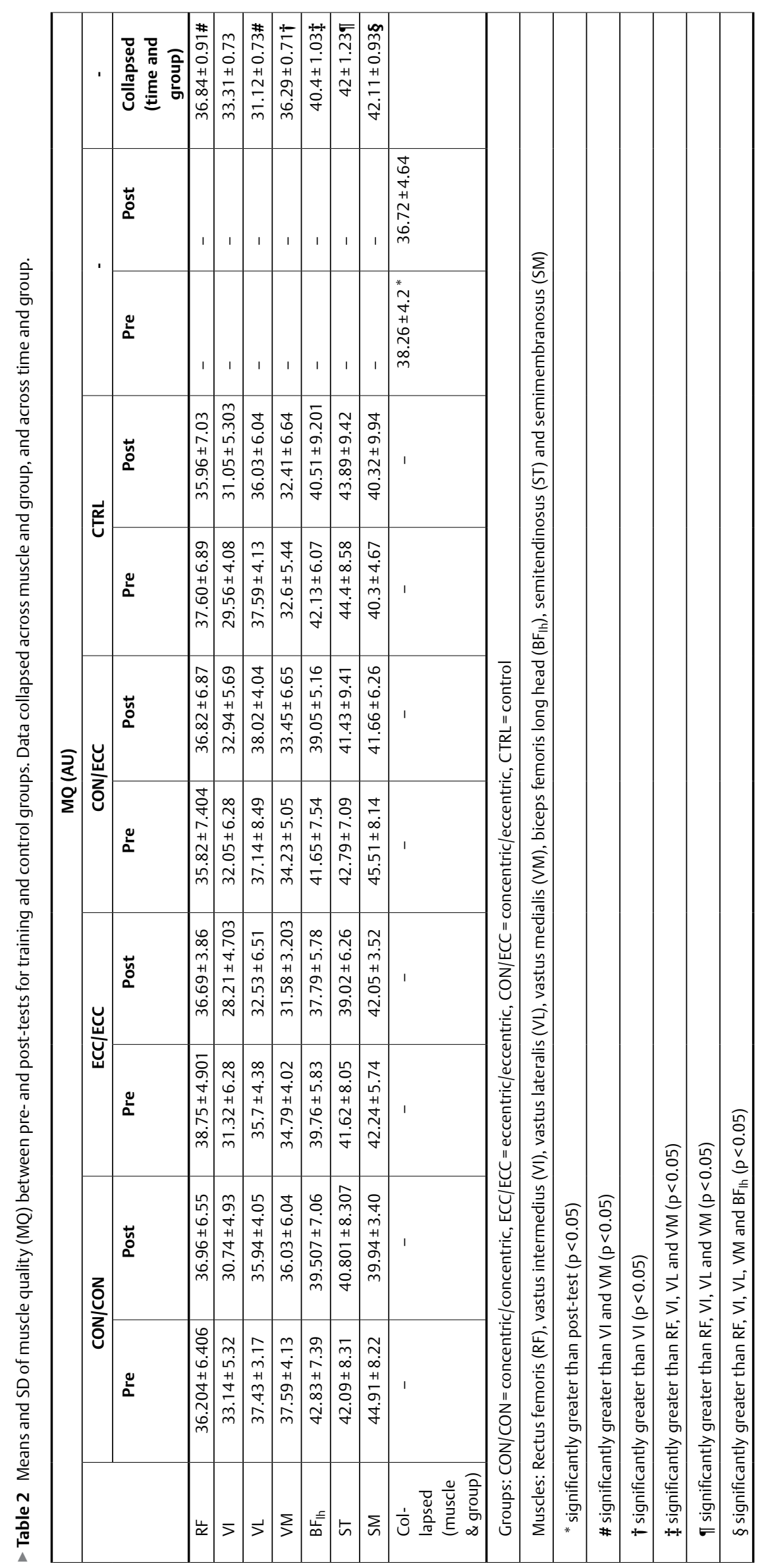




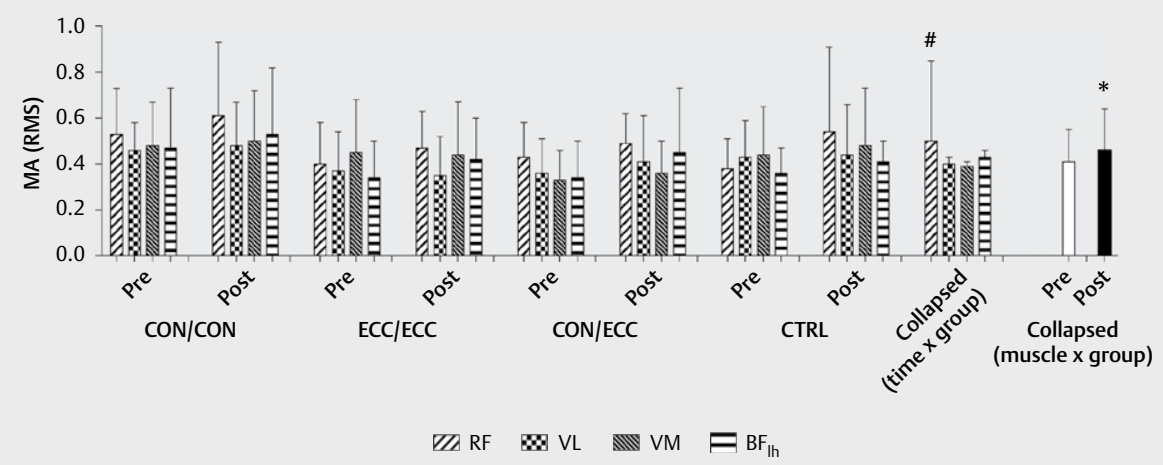

- Fig. 1 Means and SD of rectus femoris (RF), vastus lateralis (VL), vastus medialis (VM), and biceps femoris long head (BF |h $)$ muscle activation (MA) between pre- and post-tests for concentric/concentric (CON/CON), eccentric/eccentric (ECC/ECC), concentric/eccentric (CON/ECC), and control (CTRL) groups. MA was calculated by root mean square over a $1 \mathrm{~s}$ plateau of each maximal voluntary isometric contraction. Data collapsed across time and group. \# Significantly greater than VL, VM and BF $(p<0.05)$. Data collapsed across muscle and group. ${ }^{*}$ Significantly greater than pre-test $(p<0.05)$.

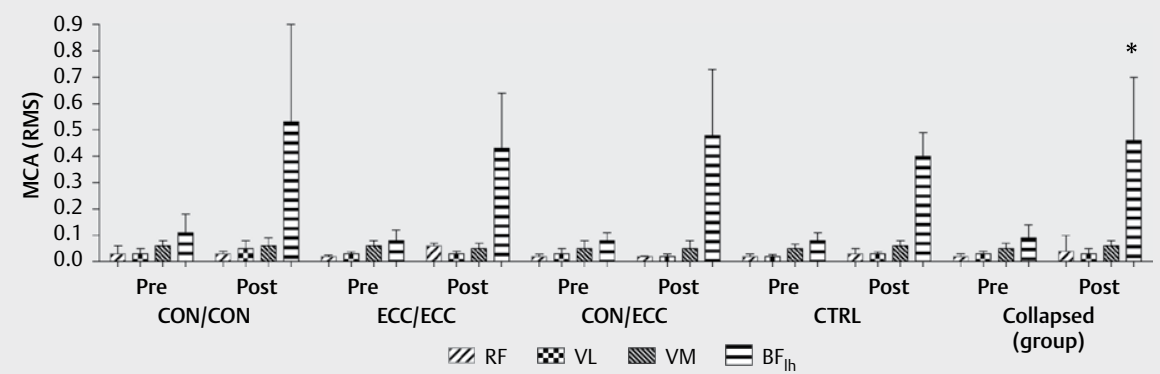

- Fig. 2 Means and SD of rectus femoris (RF), vastus lateralis (VL), vastus medialis $(\mathrm{VM})$, and biceps femoris long head $\left(\mathrm{BF}_{\mathrm{lh}}\right)$ muscle coactivation (MCA) between pre- and post-tests for concentric/concentric (CON/CON), eccentric/eccentric (ECC/ECC), concentric/eccentric. (CON/ECC), and control (CTRL) groups. MCA was calculated by root mean square over a $1 \mathrm{~s}$ plateau of each maximal voluntary isometric contraction. Data collapsed across group. * Significantly greater than $\mathrm{BF}_{\mathrm{Ih}}$ pre-test $(\mathrm{p}<0.05)$.

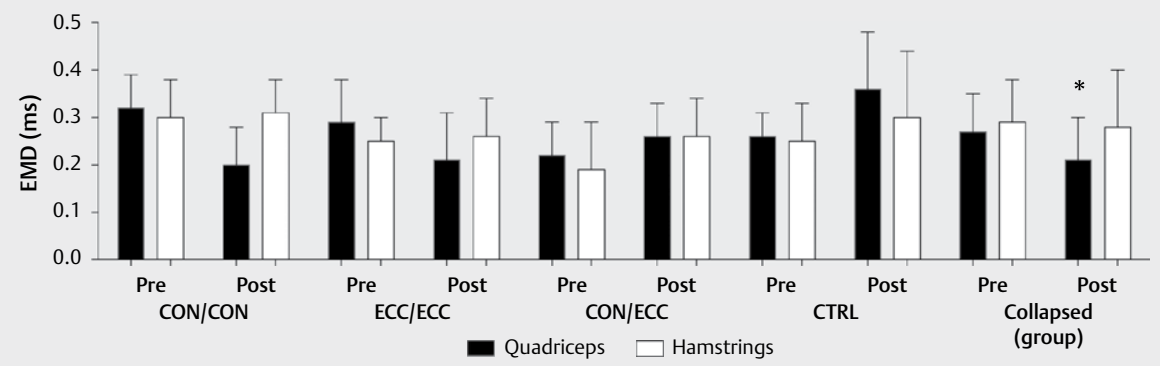

- Fig. 3 Means and SD of quadriceps and hamstrings electromechanical delay (EMD) between pre- and post-tests for concentric/concentric (CON/ CON), eccentric/eccentric (ECC/ECC), concentric/eccentric (CON/ECC), and control (CTRL) groups. Data collapsed across group. * Significantly lower than quadriceps pre-test $(p<0.05)$.

gations have demonstrated that neural adaptations may depend on differences between training and testing muscle actions $[2,4,6]$, as well as intensity [25], velocity [11,47], and duration [2] of training. Higbie et al. (1996) found that 10 weeks of maximal unilateral concentric vs. eccentric knee extensor training did not result in different MA increases. Similarly, Seger et al. [47] found that concen- tric vs. eccentric training only increased MA at similar or higher speeds than those performed during training and were not different between muscle actions. These studies are partially in agreement with our results, since we also did not find MA differences between training modes, nor did we find increases within groups. Only a few studies have investigated the effects of specific muscle action 

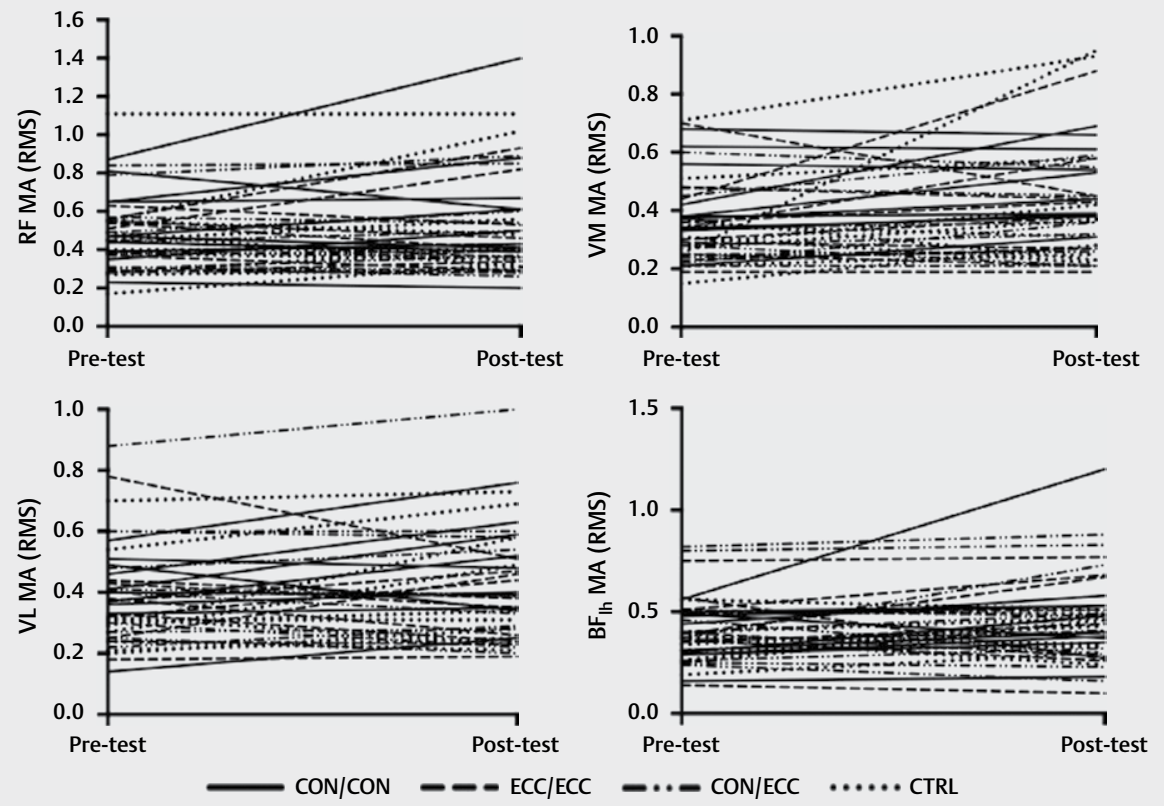

- Fig. 4 Individual data of rectus femoris (RF), vastus lateralis (VL), vastus medialis (VM), and biceps femoris long head ( $\mathrm{BF}_{\mathrm{lh}}$ ) muscle activation (MA) between pre- and post-tests for concentric/concentric (CON/CON), eccentric/eccentric (ECC/ECC), concentric/eccentric (CON/ECC), and control (CTRL) groups.
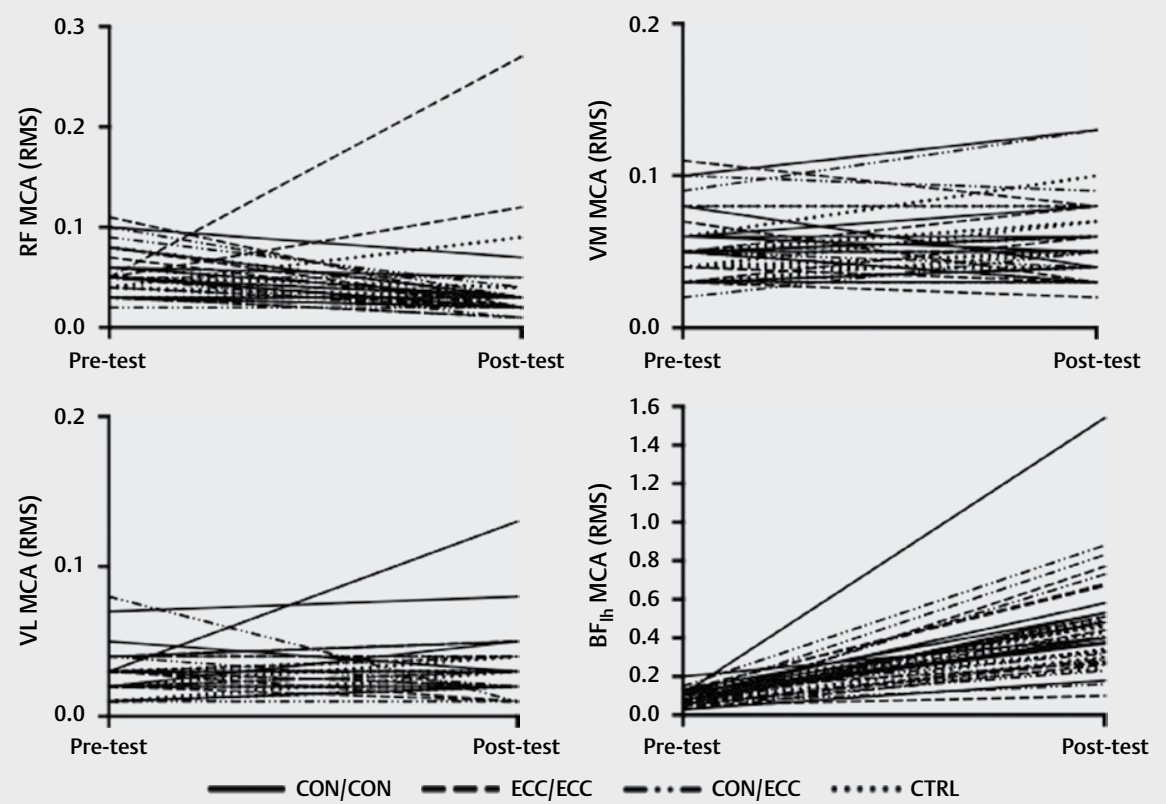

Fig. 5 Individual data of rectus femoris (RF), vastus lateralis (VL), vastus medialis (VM), and biceps femoris long head ( $\left.\mathrm{BF}_{\text {Ih }}\right)$ muscle coactivation (MCA) between pre- and post-tests for concentric/concentric (CON/CON), eccentric/eccentric (ECC/ECC), concentric/eccentric (CON/ECC), and control (CTRL) groups.

training modes on MA measured isometrically. Cadore et al. [6] found that 6 weeks of concentric vs. eccentric knee extensor training did not improve isometric MA. In addition, Baroni et al. [2] and Brassine et al. [4] were unable to find isometric MA differences after 2 and 4 weeks of specific eccentric training, although Baroni et al.
[2] found that MA adaptations measured eccentrically were present after 4 weeks. This demonstrates that MA short-term training increases may be more likely to occur when the testing is performed in the same specific muscle action as training. 

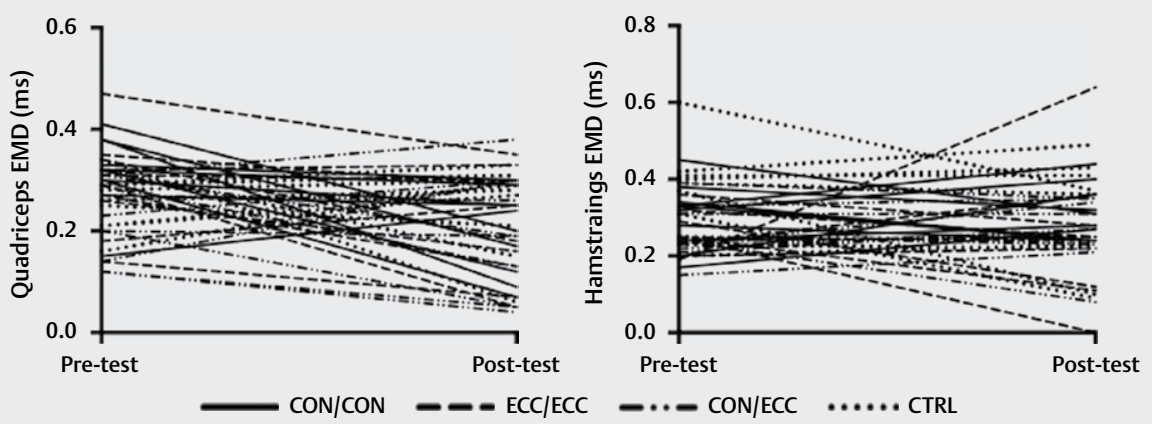

- Fig. 6 Individual data of quadriceps and hamstrings electromechanical delay (EMD) between pre- and post-tests for concentric/concentric (CON/ CON), eccentric/eccentric (ECC/ECC), concentric/eccentric (CON/ECC), and control (CTRL) groups.

Although other possible neural adaptations such as reductions in activation of synergists and antagonists may be expected after RT [24], our results indicated that MCA was unaltered between training modes. Colson et al. [13] was also unable to find MCA changes after 7 weeks of eccentric elbow flexion training. This demonstrates that short-term specific muscle action training may not be enough to modify the coactivation mechanism. Interestingly, biceps femoris long head MCA at post-test was greater than pretest when collapsed across groups. Since training may not have been sufficient to reduce MCA, it seems that maximal isometric knee extension contractions during post tests may have led the central nervous system to optimize increased co-activation for preservation of joint integrity [20]. However, biceps femoris long head MCA individual data indicate that only a few individuals in each group had greater MCA at post-test, which may have influenced these results. Nevertheless, EMG MA and MCA do not measure all possible neural adaptations due to RT, and the portion of the muscle where EMG electrodes are placed does not precisely reflect the cross sectional area of muscle fibers activated through the entire range of motion tested, nor does it account for potential differences in force generation between fiber types at different velocities [24]. Therefore, different muscle action training modes could still lead to both supraspinal and spinal specific adaptations, such as increased motoneuron excitability, reduced presynaptic inhibition, and elevated central motor drive [28]. Additionally, Ruas et al. [43] in a review reported that there are important cortical activation differences between muscle actions in strength and fatigue outcomes, which may result in specific chronic central neural adaptations.

$\mathrm{MQ}$ is commonly measured through echo-intensity, which is the greyscale quantification of muscle images $[8,44]$. Since this measurement shows muscular fat and/or fibrous composition levels, a decrease in echo-intensity values can identify improvements in muscle strength, contractile component and functionality $[7,18,38]$ due to greater muscle metabolic demand and reduction of adipose tissue $[7,37]$. We are only aware of one study that has compared vastus lateralis MQ measured by echo-intensity after specific muscle action training [6], which partially agrees with our results as they found no differences between concentric vs eccentric short-term training in MQ. Additionally, Stock et al. [48] also did not find differences in biceps brachi muscle echo-intensity measured every 72-96h during 4 weeks of concentric dumbbell curls and shoulder press resistance training. However, echo-intensity has been previously questioned as a valid single marker of MQ, since it may be affected by the variability of participants' intramuscular fat, influencing sound absorption and reflection of echo signals $[44,51]$. It may also be influenced by shape, location and size of ROI analyses of different muscles [8]. This has been shown to result in low reliability compared to other ultrasound measurements such as MT and cross sectional area [44]. Therefore, caution should be used in its interpretation as a single measurement of MQ $[8,51]$, as short-term specific muscle action RT may not be sufficient to elicit echo-intensity changes [48].

EMD reduction due to $R T$ is related to a variety of changes in the muscle-tendon complex, such as increases in contractile component, excitation-contraction coupling, and stretching of series elastic components [10, 30, 31]. Kubo et al. [30] found that 12 weeks of isometric knee extension training led to decreases in EMD associated with increases in tendon stiffness and rate of torque development. However, Costa et al. [14] did not find EMD improvements after very short isokinetic vs. dynamic constant external resistance knee extension training. Similarly, Häkkinen and Komi [22] were unable to find changes in EMD after 16 weeks of dynamic knee extensor training. Zhou [54] also did not find significant changes in knee extensor EMD after 7 weeks of sprint training. These results are in agreement with our study, as we did not find improvements in EMD for any muscle action training mode. Nevertheless, longterm weightlifting athletes have been found to have shorter EMD compared to endurance athletes [53]. Therefore, dynamic muscle action RT may require a longer time to demonstrate improvements in force transmittal capacity from the muscle-tendon complex to the skeletal system compared to isometric training. Future specific muscle action training studies, including dynamic and isometric muscle actions, are needed to further support this assumption.

In the present study, our isokinetic training progression of velocity strategy was based on the opposite force/velocity curves for concentric and eccentric muscle actions, assuming that intensity of training would be increased every week by decreasing isokinetic angular velocity for concentric and increasing it for eccentric training in $30 \%$ increments. For that reason, velocity decreased from $210 \% \mathrm{~s}-60 \% \mathrm{~s}$ for concentric training and increased from $60 \% \mathrm{~s}-210 \% \mathrm{~s}$ for eccentric training throughout the weeks. Based 
on the torque/velocity curve, if the same velocity was to be used (e. g. $90 \%$ s) then the stress would be completely different, as the concentric curve is inverse while the eccentric curve is positive [9]. This strategy was also used to ensure that groups were matched by maximal strength and theoretically similar activation levels, as when resistance training is performed maximally they belong to the same family of force/velocity curves [39]. A potential limitation of this study is that while training was performed dynamically, values for MA, MCA and EMD were measured isometrically. Therefore, it is possible that these variables might still change over time, if testing was performed specific to the muscle action trained. However, further studies are needed to clarify the specific mechanisms underpinning neuromuscular adaptations after different muscle action resistance training protocols.

\section{Conclusions}

Our findings suggest that six weeks of CON/CON, ECC/ECC or CON/ ECC muscle action isokinetic training elicit similar muscle size increases in the hamstrings and quadriceps. However, eccentric training may elicit a greater MT magnitude. Different short-term muscle action isokinetic training protocols do not lead to differential effects in MA, MCA, MQ and EMD.

\section{Conflict of Interest}

The authors declare no conflict of interest.

\section{References}

[1] Abe T, Loenneke JP, Thiebaud RS. Ultrasound assessment of hamstring muscle size using posterior thigh muscle thickness. Clin Physiol Funct Imaging 2016; 36: 206-210

[2] Baroni BM, Rodrigues R, Franke RA, Geremia JM, Rassier DE, Vaz MA. Time course of neuromuscular adaptations to knee extensor eccentric training. Int J Sports Med 2013; 34: 904-911

[3] Blazevich AJ, Cannavan D, Coleman DR, Horne S. Influence of concentric and eccentric resistance training on architectural adaptation in human quadriceps muscles. J Appl Physio 2007; 103: 1565-1575

[4] Brassine E, Mouraux D, Lambert G, Duchateau J. Effect of a short-time eccentric training on the eccentric performance of the quadriceps. Isokinet Exerc Sci 2002; 10: 23-24

[5] Brown LE, Weir JP. ASEP Procedures Recommendation I: Accurate Assessment of Muscular Strength and Power. J Exerc Physiol Online 2001; 4: 1-21

[6] Cadore EL, González-Izal M, Pallarés JG, Rodriguez-Falces J, Häkkinen K, Kraemer WJ, Pinto RS, Izquierdo M. Muscle conduction velocity, strength, neural activity, and morphological changes after eccentric and concentric training. Scand J Med Sci Sports 2014; 24: 343-352

[7] Cadore EL, Izquierdo M, Alberton CL, Pinto RS, Conceição M, Cunha G, Radaelli R, Bottaro M, Trindade GT, Kruel LFM. Strength prior to endurance intra-session exercise sequence optimizes neuromuscular and cardiovascular gains in elderly men. Exp Gerontol 2012; 47: 164-169
[8] Caresio C, Molinari F, Emanuel G, Minetto MA. Muscle echo intensity: reliability and conditioning factors. Clin Physiol Funct Imaging 2015; 35: 393-403

[9] Carney KR, Brown LE, Coburn JW, Spiering BA, Bottaro M. Eccentric torque-velocity and power-velocity relationships in men and women. Eur J Sport Sci 2012; 12: 139-144

[10] Cavanagh PR, Komi PV. Electromechanical delay in human skeletal muscle under concentric and eccentric contractions. Eur J Appl Physiol 1979; 42: 159-163

[11] Coburn JW, Housh TJ, Malek MH, Weir JP, Cramer JT, Beck TW, Johnson GO. Neuromuscular responses to three days of velocity-specific isokinetic training. J Strength Cond Res 2006; 20: 892-898

[12] Colliander EB, Tesch PA. Effects of eccentric and concentric muscle actions in resistance training. Acta Physiol Scand 1990; 140: 31-39

[13] Colson S, Pousson M, Martin A, Van Hoecke J. Isokinetic elbow flexion and coactivation following eccentric training. J Electromyogr Kinesiol 1999; 9: 13-20

[14] Costa PB, Herda TJ, Walter AA, Valdez AM, Cramer JT. Effects of short-term resistance training and subsequent detraining on the electromechanical delay. Muscle Nerve 2013; 48: 135-136

[15] Day S. Important factors in surface EMG measurement. InBortec Biomedical Ltd publishers; 2002

[16] De Lisio M, Farup J, Sukiennik RA, Clevenger N, Nallabelli J, Nelson B, Ryan K, Rahbek SK, de Paoli F, Vissing K. The acute response of pericytes to muscle-damaging eccentric contraction and protein supplementation in human skeletal muscle. J Appl Physio 2015; 119: 900-907

[17] Ferri-Morales A, Alegre LM, Basco A, Aguado X. Test-retest relative and absolute reliability of knee extensor strength measures and minimal detectable change. Isokinet Exerc Sci 2014; 22: 17-26

[18] Fragala MS, Kenny AM, Kuchel GA. Muscle quality in aging: a multi-dimensional approach to muscle functioning with applications for treatment. Sports Med 2015; 45: 641-658

[19] Franchi MV, Atherton PJ, Reeves ND, Flück M, Williams J, Mitchell WK, Selby A, Beltran Valls RM, Narici MV. Architectural, functional and molecular responses to concentric and eccentric loading in human skeletal muscle. Acta Physiol (Oxf) 2014; 210: 642-654

[20] Gabriel DA, Kamen G, Frost G. Neural adaptations to resistive exercise: mechanisms and recommendations for training practices. Sports Med 2006; 36: 133-149

[21] Golik-Peric D, Drapsin M, Obradovic B, Drid P. Short-term isokinetic training versus isotonic training: Effects on asymmetry in strength of thigh muscles. J Hum Kinet 2011; 30: 29-35

[22] Häkkinen K, Komi PV. Changes in neuromuscular performance in voluntary and reflex contraction during strength training in man. Int J Sports Med 1983; 4: 282-288

[23] Harriss D], Macsween A, Atkinson G. Standards for ethics in sport and exercise science research: 2018 Update. Int J Sports Med 2017; 38: $1126-1131$

[24] Higbie EJ, Cureton KJ, Warren GL 3rd, Prior BM. Effects of concentric and eccentric training on muscle strength, cross-sectional area, and neural activation. J Appl Physio (1985) 1996; 81: 2173-2181

[25] Jenkins NDM, Miramonti AA, Hill EC, Smith CM, Cochrane-Snyman KC, Housh TJ, Cramer JT. Greater Neural Adaptations following High- vs. Low-Load Resistance Training. Front Physiol 2017; 8: 331

[26] Jenkins NDM, Palmer TB, Cramer JT. Comparing the reliability of voluntary and evoked muscle actions. Clin Physiol Funct Imaging 2014; 34: 434-441

[27] Katsavelis D, Threlkeld AJ. Quantifying thigh muscle co-activation during isometric knee extension contractions: Within-and betweensession reliability. J Electromyogr Kinesiol 2014; 24: 502-507 
[28] Kidgell DJ, Pearce AJ. What has transcranial magnetic stimulation taught us about neural adaptations to strength training? A brief review. J Strength Cond Res 2011; 25: 3208-3217

[29] Kovaleskji JE, Heitman RJ. Testing and training the lower extremity. In: Brown L.ed. Isokinetics in human performance. Champaign, IL: Human Kinetics; 2000: 171-195

[30] Kubo K, Kanehisa H, Ito M, Fukunaga T. Effects of isometric training on the elasticity of human tendon structures in vivo. J Appl Physio (1985) 2001; 91: 26-32

[31] Lacourpaille L, Hug F, Nordez A. Influence of passive muscle tension on electromechanical delay in humans. PloS one 2013; 8: e53159

[32] Munro BH. Statistical methods for health care research. 5th ed.Philadelphia, PA: Lippincott Williams \& Wilkins; 2005

[33] Negrete R], Schick EA, Cooper JP. Lower-limb dominance as a possible etiologic factor in noncontact anterior cruciate ligament tears. J Strength Cond Res 2007; 21: 270-273

[34] Nosaka K, Sakamoto K, Newton M, Sacco P. How long does the protective effect on eccentric exercise-induced muscle damage last? Med Sci Sports Exerc 2001; 33: 1490-1495

[35] Ogborn D, Schoenfeld B]. The role of fiber types in muscle hypertrophy: implications for loading strategies. Strength Cond J 2014; 36 : 20-25

[36] Palmer TB, Akehi K, Thiele RM, Smith DB, Thompson B]. Reliability of panoramic ultrasound imaging in simultaneously examining muscle size and quality of the hamstring muscles in young, Healthy Males and Females. Ultrasound Med Biol 2015; 41: 675-684

[37] Pillen S, Tak RO, Zwarts M], Lammens MMY, Verrijp KN, Arts IMP, van der Laak JA, Hoogerbrugge PM, van Engelen BGM, Verrips A. Skeletal muscle ultrasound: Correlation between fibrous tissue and echo intensity. Ultrasound Med Biol 2009; 35: 443-446

[38] Rech A, Radaelli R, Goltz FR, da Rosa LHT, Schneider CD, Pinto RS. Echo intensity is negatively associated with functional capacity in older women. Age 2014; 36: 1-9

[39] Reeves ND, Maganaris CN, Longo S, Narici MV. Differential adaptations to eccentric versus conventional resistance training in older humans. Exp Physiol 2009; 94: 825-833

[40] Reid S, Hamer P, Alderson J, Lloyd D. Neuromuscular adaptations to eccentric strength training in children and adolescents with cerebral palsy. Dev Med Child Neurol 2010; 52: 358-363

[41] Rhea MR. Determining the magnitude of treatment effects in strength training research through the use of the effect size. J Strength Cond Res 2004; 18: 918-920
[42] Ruas CV, Brown LE, Lima CD, Costa PB, Pinto RS. Effect of three different muscle action training protocols on knee strength ratios and performance. J Strength Cond Res 2017 doi:10.1519/JSC.0000000000002134 Epub ahead of print

[43] Ruas CV, Lima CD, Pinto RS, Oliveira MA, Barros JAC, Brown LE. Brain activation differences between muscle actions for strength and fatigue: A brief review. Braz J Mot Behav 2016; 10: 1-8

[44] Ruas CV, Pinto RS, Lima CD, Costa PB, Brown LE. Test-retest reliability of muscle thickness, echo-intensity and cross sectional area of quadriceps and hamstrings muscle groups using B-mode ultrasound. Int J Kines Sport Sci 2017; 5:

[45] Schoenfeld BJ. The mechanisms of muscle hypertrophy and their application to resistance training. J Strength Cond Res 2010; 24: 2857-2872

[46] Schoenfeld B], Ogborn D, Krieger JW. Effects of resistance training frequency on measures of muscle hypertrophy: A systematic review and meta-analysis. Sports Med 2016; 46: 1689-1697

[47] Seger JY, Arvidsson B, Thorstensson A. Specific effects of eccentric and concentric training on muscle strength and morphology in humans. Eur J Appl Physiol 1998; 79: 49-57

[48] Stock MS, Olinghouse KD, Mota JA, Drusch AS, Thompson BJ. Muscle group specific changes in the electromechanical delay following short-term resistance training. J Sci Med Sport 2016; 19: 761-765

[49] Weir JP. Quantifying test-retest reliability using the intraclass correlation coefficient and the SEM. J Strength Cond Res 2005; 19: 231-240

[50] Wernbom M, Augustsson J, Thomeé R. The influence of frequency, intensity, volume and mode of strength training on whole muscle cross-sectional area in humans. Sports Med 2007; 37: 225-264

[51] Young H], Jenkins NT, Zhao Q, McCully KK. Measurement of intramuscular fat by muscle echo intensity. Muscle Nerve 2015; 52: 963-971

[52] Yu JY, Jeong JG, Lee BH. Evaluation of muscle damage using ultrasound imaging. J Phys Ther Sci 2015; 27: 531-534

[53] Zhou S. Electromechanical delay in weight lifters and endurance trained athletes. XVth Congress of the International Society of Biomechanics: book of abstracts. 1995; Jyvaskyla, Finland: 1036-1037

[54] Zhou S, McKenna M], Lawson DL, Morrison WE, Fairweather I. Effects of fatigue and sprint training on electromechanical delay of knee extensor muscles. Eur J Appl Physiol 1996; 72: 410-416 